die tDCS, ebenso wie andere Therapieverfahren, eine multimodale Behandlung nicht ersetzen kann. Ihre leichte Handhabbarkeit, vergleichsweise geringe Behandlungskosten und gute Verträglichkeit sind allerdings zweifellos Stärken. Neben der Durchführung weiterer hochwertiger klinischer Studien zur Wirksamkeit der verschiedenen Methoden und Parameter stellt sich in Zukunft die Herausforderung der adäquaten Einbindung transkranieller Hirnstimulation in die psychiatrisch-psychotherapeutische Versorgung, um diese neuen Möglichkeiten im Sinne der Patienten adäquat auszuschöpfen.

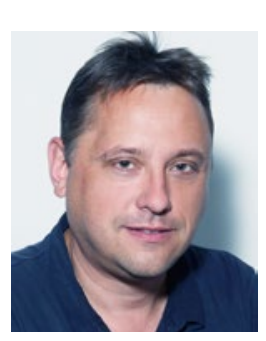

Prof. Dr. med. Christian Plewnia, Tübingen

Klinik für Psychiatrie und Psychotherapie, Neurophysiologie \& interventionelle

Neuropsychiatrie, Tübingen

E-Mail: christian.plewnia@uni-tuebingen.de

\title{
Welches Schmerzmittel bei Arthrose?
}

Fragestellung: Welche nicht steroidalen Antirheumatika (NSAR) sind bei der Behandlung der Hüft- und Kniegelenksarthrose am besten wirksam und haben das beste Sicherheitsprofil?

Hintergrund: Mit zunehmendem Alter steigt die Häufigkeit von Arthrose und Arthritis an Hüft- und Kniegelenken. Basis der medikamentösen Therapie sind Analgetika und NSAR, die zumindest so lange eingesetzt werden, bis ein operativer Gelenkersatz notwendig wird. Da sich in der Neurologie viele ältere $\mathrm{Pa}$ tienten befinden, die unter diesen Beschwerden leiden, ist insbesondere unter den Aspekten der Komedikation die Information wichtig, welche Medikamente hier am besten wirksam sind.

Patienten und Methodik: Im Rahmen einer Netzwerkmetaanalyse wurden randomisierte Studien analysiert, die NSAR, Paracetamol oder Placebo für die Behandlung von Knie- und

Da Costa BR, Reichenbach S, Keller $\mathrm{N}$ et al. Effectiveness of non-steroidal anti-inflammatory drugs for the treatment of pain in knee and hip osteoarthritis: a network meta-analysis. Lancet 2017; 390: e21-e33 Hüftgelenksschmerzen untersucht hatten. Eingeschlossen wurden Studien, die zwischen 1980 und Februar 2015 durchgeführt wurden. Die klinischen Endpunkte waren Schmerz und Funktion.
Ergebnisse: Die Autoren identifizierten 76 randomisierte Studien mit insgesamt 58.451 Patienten, die die Einschlusskriterien für eine Metaanalyse erfüllten. Alle untersuchten Analgetika waren einer Behandlung mit Placebo für den Endpunkt Schmerzintensität überlegen. Die weitere Auswertung erfolgte dahingehend, ob ein minimaler, klinisch bedeutsamer Unterschied zwischen den Studien bestand. Dies war definiert als 0,37 Standardabweichungen gegenüber Placebo. Für Diclofenac 150 mg/Tag, Etoricoxib 30, 60 und 90 mg/Tag und Rofecoxib 25 und $50 \mathrm{mg} / \mathrm{Tag}$ fand sich eine klinisch bedeutsame Reduktion der Schmerzen im Vergleich zu Placebo. Der Vertrieb von Rofecoxib wurde aber in Deutschland 2004 eingestellt, da es unter der Therapie zu einer Zunahme kardiovaskulärer Endpunkte gekommen war. Die Wirksamkeit der untersuchten NSAR nahm mit der Dosis zu, wobei sich eine lineare Beziehung nur für Naproxen fand. Eine Untersuchung über längere Zeiträume ergab keinen Hinweis darauf, dass NSAR an Wirksamkeit verlieren. Paracetamol war in der Metaanalyse nur in sehr hohen Tagesdosen von $4.000 \mathrm{mg}$ einer Behandlung mit Placebo überlegen.

Schlussfolgerungen: Paracetamol war zur Schmerztherapie bei Patienten mit Knie- und Hüftgelenksarthrose einer Behandlung mit Placebo nicht überlegen. In der Gruppe der NSAR war Diclofenac in einer Dosis von $150 \mathrm{mg} / \mathrm{Tag}$ am wirksamsten.

\section{- Kommentar von Hans Christoph Diener, Essen}

\section{Diclofenac und Etoricoxib am wirksamsten bei Gelenkschmerzen}

Diese große Metaanalyse zeigt bei Gelenkschmerzen durch Arthrose genauso wie bei chronischen Rückenschmerzen, dass Paracetamol entweder nicht wirksam ist oder in so hohen Dosen gegeben werden muss, dass langfristig eine Beeinträchtigung der Leberfunktion zu befürchten ist. Unter den nicht steroidalen Antirheumatika waren Etoricoxib und Diclofenac am besten wirksam.

In letzter Zeit hat sich auch die Diskussion über die potenziell gefährlichen Eigenschaften der Coxibe bezüglich kardiovaskulärer Erkrankungen relativiert. Eine große randomisierte Studie in den USA hatte keinen Hinweis auf ein erhöhtes Risiko kardiovaskulärer Ereignisse für Celecoxib gezeigt (PRECISIONStudie) [1]. Außerdem erhöhen offenbar sowohl Coxibe als auch NSAR das Risiko für kardiovaskuläre Erkrankungen. Dieses Risiko muss allerdings abgemildert werden, wenn man berücksichtigt, dass sich unbehandelte Patienten mit Schmerzen weniger bewegen können und damit Bewegung und Sport als präventive kardiovaskuläre Maßnahmen entfallen.

\footnotetext{
Referenz

1. Nissen SE et al. N Engl J Med 2016; 375: 2519-29
} 